\title{
Fractional Boson Gas and Fractional Poisson Measure in Infinite Dimensions
}

\author{
Maria João Oliveira and Rui Vilela Mendes
}

\begin{abstract}
As a consequence of Haag's theorem, to obtain a non-trivial theory, one either works with a non-Fock representation or with a Fock representation in a finite volume. Calculations in the Fock representation taking the $\mathrm{N}, \mathrm{V} \rightarrow \infty$ limit with the ratio $\mathrm{N} / \mathrm{V}=\rho$ fixed, show the equivalence of the free Boson gas and the infinitedimensional Poisson measure. The N/V limit provides a way to deal with non-trivial infinite systems using the Fock representation. However, by the very nature of the fixed $\rho$ density limit, it is unable to deal with systems with density fluctuations, a shortcoming that is solved by the use of reducible functionals. A particularly interesting reducible functional is the one associated to the infinite-dimensional fractional Poisson measure which we recall in this work.
\end{abstract}

Keywords Boson gases · Fractional Poisson measure

\section{Introduction: Density Fluctuations, Reducible Functionals and Fractional Gases}

Systems with an infinite number of degrees of freedom have, in addition to the Fock representation, infinitely many inequivalent representations of the canonical commutation relations. Haag's theorem states that, in a theory with a space-invariant vacuum, any representation equivalent to Fock can only describe a free system. Therefore, to obtain a non-trivial theory, one either works with a non-Fock representation or with a Fock representation in a finite volume. In this latter case one considers $N$ particles in a finite volume $V$. Calculations are carried out in the Fock representation and in

\footnotetext{
M.J. Oliveira

Universidade Aberta and Centro de Matemática e Aplicações Fundamentais,

Lisbon, Portugal

e-mail: oliveira@cii.fc.ul.pt

R.V. Mendes ( $\square)$

Centro de Matemática e Aplicações Fundamentais, Lisbon, Portugal

e-mail: rvmendes@fc.ul.pt; rvilela.mendes@gmail.com 
the end one takes $N, V \rightarrow \infty$ with the ratio $N / V=\rho$ being fixed. Thus, the $N / V$ limit provides a way to deal with non-trivial infinite systems using the Fock representation. However, by the very nature of the fixed $\rho$ density limit, this approach is unable to deal with systems with density fluctuations. This shortcoming is solved by the use of the reducible functionals to be described later on.

First, let us recall the connection of the infinite-dimensional Poisson measure to the free Bose gas. A particularly convenient way to establish this connection, and also to explore generalizations, is the framework of non-relativistic current algebra of many-body systems. That is, the basic variables of the many-body system are the smeared currents $[1,2]$ (see also $[3,4]$ and references therein)

$$
\begin{aligned}
& \rho(f)=\int d^{3} x f(x) \rho(x) \\
& \mathbf{J}(\mathbf{g})=\int d^{3} x \mathbf{J}(x) \bullet \mathbf{g}(x)
\end{aligned}
$$

$f$ and $\mathbf{g}$ being respectively smooth compact support functions and vector fields. The smeared currents satisfy the infinite-dimensional Lie algebra,

$$
\begin{aligned}
& {[\rho(f), \rho(h)]=0} \\
& {[\rho(f), \mathbf{J}(\mathbf{g})]=i \rho(\mathbf{g} \bullet \nabla f)} \\
& {[\mathbf{J}(\mathbf{g}), \mathbf{J}(\mathbf{k})]=i \mathbf{J}(\mathbf{k} \bullet \nabla \mathbf{g}-\mathbf{g} \bullet \nabla \mathbf{k})}
\end{aligned}
$$

each particular physical system corresponding to a different Hilbert space representation of this algebra or of the semidirect product group generated by the exponentiated currents

$$
\begin{aligned}
U(f) & =e^{i \rho(f)} \\
V\left(\phi_{t}^{\mathbf{g}}\right) & =e^{i \mathbf{J}(\mathbf{g})}
\end{aligned}
$$

$\phi_{t}^{\mathbf{g}}$ being the flow of the vector field $\mathbf{g}$

$$
\frac{d}{d t} \phi_{t}^{\mathbf{g}}(x)=\mathbf{g}\left(\phi_{t}^{\mathbf{g}}(x)\right)
$$

For a system of $N$ free bosons in a box of volume $V$, the normalized ground state is

$$
\Omega_{N, V}\left(x_{1}, \cdots, x_{N}\right)=\left(\frac{1}{\sqrt{V}}\right)^{N}
$$

and the ground state functional

$$
L_{N, V}(f)=\left(\Omega_{N, V}, U_{N, V}(f) \Omega_{N, V}\right)
$$




$$
=\frac{1}{V}\left(\int_{V} d^{3} x e^{i f(x)}\right)^{N}
$$

In general, this functional determines not only the representation of $U(f)$ but also that of $V\left(\phi_{t}^{\mathbf{g}}\right)$, up to a complex phase multiplier.

In the $N \rightarrow \infty$ limit with constant average density $\bar{\rho}=\frac{N}{V}$ (also called the $N / V$ limit) one obtains

$$
\begin{aligned}
L(f) & =\lim _{n \rightarrow \infty}\left(1+\frac{\bar{\rho}}{N} \int\left(e^{i f(x)}-1\right) d^{3} x\right)^{n} \\
& =\exp \left(\int\left(e^{i f(x)}-1\right) \bar{\rho} d^{3} x\right)
\end{aligned}
$$

which one recognizes as the characteristic functional of the infinite-dimensional Poisson measure. Identifying $\bar{\rho} d^{3} x$ as the measure $d \mu$ in the underlying space $M$ (see Appendix 1), the $L$ functional may also be written as a vacuum expectation functional. Expanding the exponential in (1)

$$
L(f)=\sum_{n=0}^{\infty} \frac{e^{-\int_{V} d \mu}}{n !}\left(\int_{V} e^{i f(x)} d \mu\right)^{n}
$$

one may write

$$
L(f)=(\Omega, U(f) \Omega)
$$

for

$$
\Omega=\bigoplus_{n} e^{-\frac{1}{2} \int_{V} d \mu} 1_{n}
$$

$1_{n}$ denotes the identity function in the $n$-particle space and the $\frac{1}{n !}$ factor in (2) is recovered by the symmetrization operation.

The conclusion is that an infinite dimensional free Boson gas at constant density $\bar{\rho}$ is completely characterized by the infinite-dimensional Poisson measure. However (1) is not the most general consistent representation of the nonrelativistic current algebra, a more general one being [2]

$$
L(f)=\int_{0}^{\infty} \exp \left(\bar{\rho} \int\left(e^{i f(x)}-1\right) d^{3} x\right) d \mu(\bar{\rho})
$$

with $\mu$ a positive measure on $[0, \infty)$ normalized so that $\int_{0}^{\infty} d \mu(\bar{\rho})=1$.

Physically this reducible functional represents a Boson gas with density fluctuations. Among the many possible reducible functionals consistent with (3) there is a fractional generalization of (2), namely 


$$
L_{\alpha}(f)=\sum_{n=0}^{\infty} \frac{E_{\alpha}^{(n)}\left(-\int_{V} d \mu\right)}{n !}\left(\int_{V} e^{i f(x)} d \mu\right)^{n}
$$

$(0<\alpha \leq 1)$, which corresponds to a vacuum state

$$
\Omega_{\alpha}=\bigoplus_{n} \sqrt{E_{\alpha}^{(n)}\left(-\int_{V} d \mu\right)} 1_{n}
$$

$E_{\alpha}^{(n)}$ denoting the $n$-th derivative of the Mittag-Leffler function [5]. $\Omega_{\alpha}$ differs from $\Omega$ in the weight given to each one of the $n$-particle spaces.

The measure associated to the functional (4) is called the infinite-dimensional fractional Poisson measure and the corresponding physical system the fractional Boson gas.

In the same way as the infinite-dimensional Poisson measure completely characterizes the Boson gas, the infinite-dimensional fractional Poisson measure will characterize a "fractional Boson gas" and the remainder of this work is dedicated to recall the main properties of this measure [6]. A detailed analysis of the mathematical properties of the fractional Poisson measure is a precondition for the rigorous formulation of some of the already explored physical implications of fractality (see for example $[7,8])$.

\section{The Infinite-Dimensional Fractional Poisson Measure}

\subsection{The Fractional Poisson Process}

The Poisson measure $\pi$ in $\mathbb{R}($ or $\mathbb{N}$ ) is

$$
\pi(A)=e^{-\sigma} \sum_{n \in A} \frac{\sigma^{n}}{n !}
$$

the parameter $\sigma$ being called the intensity. The Laplace transform of $\pi$ is

$$
l_{\pi}(\lambda)=\mathbb{E}\left(e^{\lambda \cdot}\right)=e^{-\sigma} \sum_{n=0}^{\infty} \frac{\sigma^{n}}{n !} e^{\lambda n}=e^{\sigma\left(e^{\lambda}-1\right)}
$$

For $n$-tuples of independent Poisson variables one would have

$$
l_{\pi}(\lambda)=e^{\sum \sigma_{k}\left(e^{\lambda} k-1\right)}
$$

Continuing $\lambda_{k}$ to imaginary arguments $\lambda_{k}=i f_{k}$, the characteristic function is 


$$
C_{\pi}(\lambda)=e^{\sum \sigma_{k}\left(e^{i f_{k}}-1\right)}
$$

Looked at as a renewal process, $P(X=n)=e^{-\sigma} \frac{\sigma^{n}}{n !}$ would be the probability of $n$ events occurring in the time interval $\sigma$. The survival probability, that is, the probability of no event is

$$
\Psi(\sigma)=e^{-\sigma}
$$

which satisfies the equation

$$
\frac{d}{d \sigma} \Psi(\sigma)=-\Psi(\sigma)
$$

Replacing in (6) the derivative $\frac{d}{d \sigma}$ by the (Caputo) fractional derivative

$$
D^{\alpha} \Psi(\sigma)=\frac{1}{\Gamma(1-\alpha)} \int_{0}^{\sigma} \frac{\Psi^{\prime}(\tau)}{(\sigma-\tau)^{\alpha}} d \tau=-\Psi(\sigma) \quad(0<\alpha<1)
$$

one has the solution

$$
\Psi(\sigma)=E_{\alpha}\left(-\sigma^{\alpha}\right)
$$

with $E_{\alpha}$ being the Mittag-Leffler function of parameter $\alpha$

$$
E_{\alpha}(z)=\sum_{n=0}^{\infty} \frac{z^{n}}{\Gamma(\alpha n+1)}, \quad z \in \mathbb{C}
$$

$(\alpha>0)$. One then obtains a fractional Poisson process $[9,10]$ with the probability of $n$ events

$$
P(X=n)=\frac{\sigma^{\alpha n}}{n !} E_{\alpha}^{(n)}\left(-\sigma^{\alpha}\right)
$$

$E_{\alpha}^{(n)}$ denoting the $n$-th derivative of the Mittag-Leffler function. In contrast with the Poisson case $(\alpha=1)$, this process has power law asymptotics rather than exponential, which implies that it is not anymore Markovian. The characteristic function of this process is given by

$$
C_{\alpha}(\lambda)=E_{\alpha}\left(\sigma^{\alpha}\left(e^{i \lambda}-1\right)\right)
$$

\subsection{The Infinite-Dimensional Fractional Poisson Measure}

For the Poisson measure $(\alpha=1)$ an infinite-dimensional generalization is obtained by generalizing (5) to

$$
C(\varphi)=e^{\int\left(e^{i \varphi(x)}-1\right) d \mu(x)}
$$


for test functions $\varphi \in \mathscr{D}(M), \mathscr{D}(M)$ being the space of $C^{\infty}$-functions of compact support in a manifold $M$ (fixed from the very beginning), and then using the Bochner-Minlos theorem to show that $C$ is the Fourier transform of a measure on the distribution space $\mathscr{D}^{\prime}(M)$. Because the Mittag-Leffler function is a natural analytic generalization of the exponential function one conjectures that an infinitedimensional version of the fractional Poisson measure would have a characteristic functional

$$
C_{\alpha}(\varphi):=E_{\alpha}\left(\int\left(e^{i \varphi(x)}-1\right) d \mu(x)\right), \quad \varphi \in \mathscr{D}(M)
$$

with $\mu$ a positive intensity measure fixed on the underlying manifold $M$. However, a priori it is not obvious that this is the Fourier transform of a measure on $\mathscr{D}^{\prime}(M)$ nor that it corresponds to independent processes because the Mittag-Leffler function does not satisfy the factorization properties of the exponential.

Similarly to the Poisson case, to carry out our construction and analysis in detail we always assume that $M$ is a geodesically complete connected oriented (non-compact) Riemannian $C^{\infty}$-manifold, where we fix the corresponding Borel $\sigma$-algebra $\mathscr{B}(M)$, and $\mu$ is a non-atomic Radon measure, which we assume to be non-degenerate (i.e., $\mu(O)>0$ for all non-empty open sets $O \subset M)$. Having in mind the most interesting applications, we also assume that $\mu(M)=\infty$.

Theorem 1 For each $0<\alpha \leq 1$ fixed, the functional $C_{\alpha}$ in Eq. (8) is the characteristic functional of a probability measure $\pi_{\mu}^{\alpha}$ on the distribution space $\mathscr{D}^{\prime}(M)$.

Proof That $C_{\alpha}$ is continuous and $C_{\alpha}(0)=1$ follows easily from the properties of the Mittag-Leffler function. To check the positivity one uses the complete monotonicity of $E_{\alpha}, 0<\alpha<1$, which by Appendix 2 (Lemma 1) implies the integral representation

$$
E_{\alpha}(-z)=\int_{0}^{\infty} e^{-\tau z} d v_{\alpha}(\tau)
$$

for any $z \in \mathbb{C}$ such that $\operatorname{Re}(z) \geq 0, v_{\alpha}$ being the probability measure (19) (Appendix 2). Hence by (9)

$$
\sum_{a, b} C_{\alpha}\left(\varphi_{a}-\varphi_{b}\right) z_{a}^{*} z_{b}=\int_{0}^{\infty} d v_{\alpha}(\tau) \sum_{a, b} e^{-\tau \int_{M} d \mu(x)\left(1-e^{i\left(\varphi_{a}-\varphi_{b}\right)}\right)} z_{a}^{*} z_{b}
$$

Each one of the terms in the integrand corresponds to the characteristic function of a Poisson measure. Thus, for each $\tau$ the integrand is positive and therefore the spectral integral (10) is also positive. From the Bochner-Minlos theorem it then follows that $C_{\alpha}$ is the characteristic functional of a probability measure $\pi_{\mu}^{\alpha}$ on the measurable space $\left(\mathscr{D}^{\prime}(M), \mathscr{C}_{\sigma}\left(\mathscr{D}^{\prime}(M)\right)\right), \mathscr{C}_{\sigma}\left(\mathscr{D}^{\prime}(M)\right)$ being the $\sigma$-algebra generated by the cylinder sets.

For $\alpha=1$ see e.g. [11]. 
Introducing the fractional Poisson measure by the above approach yields a probability measure on $\left(\mathscr{D}^{\prime}(M), \mathscr{C}_{\sigma}\left(\mathscr{D}^{\prime}(M)\right)\right)$. The next step is to find an appropriate support for the fractional Poisson measure. Using the analyticity of the Mittag-Leffler function one may informally rewrite (8) as

$$
\begin{aligned}
C_{\alpha}(\varphi) & =\sum_{n=0}^{\infty} \frac{E_{\alpha}^{(n)}\left(-\int d \mu(x)\right)}{n !}\left(\int e^{i \varphi(x)} d \mu(x)\right)^{n} \\
& =\sum_{n=0}^{\infty} \frac{E_{\alpha}^{(n)}\left(-\int d \mu(x)\right)}{n !} \int e^{i\left(\varphi\left(x_{1}\right)+\varphi\left(x_{2}\right)+\cdots+\varphi\left(x_{n}\right)\right)} d \mu^{\otimes n}
\end{aligned}
$$

For the Poisson case $(\alpha=1)$ instead of $E_{\alpha}^{(n)}\left(-\int d \mu(x)\right)$ one would have exp $\left(-\int d \mu(x)\right)$ for all $n$, the rest being the same, cf. Appendix 1. Therefore one concludes that the main difference in the fractional case $(\alpha \neq 1)$ is that a different weight is given to each $n$-particle space, but that a configuration space [12-14] is also the natural support of the fractional Poisson measure. The explicit construction is made below.

Notice however that the different weights, multiplying the $n$-particle space measures, are physically quite significant in that they have decays, for large volumes, much smaller than the corresponding exponential factor in the Poisson measure.

Using now the spectral representation (9) of the Mittag-Leffler function one may rewrite (8) as

$$
C_{\alpha}(\varphi)=\int_{0}^{\infty} \exp \left(\tau \int\left(e^{i \varphi(x)}-1\right) d \mu(x)\right) d \nu_{\alpha}(\tau)
$$

with the integrand being the characteristic function of the Poisson measure $\pi_{\tau \mu}$, $\tau>0$. In other words, the characteristic functional (8) coincides with the characteristic functional of the measure $\int_{0}^{\infty} \pi_{\tau \mu} d v_{\alpha}(\tau)$. By uniqueness, this implies the integral decomposition

$$
\pi_{\mu}^{\alpha}=\int_{0}^{\infty} \pi_{\tau \mu} d v_{\alpha}(\tau)
$$

meaning that $\pi_{\mu}^{\alpha}$ is an integral (or mixture) of Poisson measures $\pi_{\tau \mu}, \tau>0$. The measure $d \nu_{\alpha}(\tau)$ corresponds to the measure $d \mu(\bar{\rho})$ in Eq. (3), defining the particular reducible functional that characterizes the fractional Boson gas.

\subsection{The Fractional Poisson Measure on $\Gamma$}

The fractional Poisson measure has support in the configuration space $\Gamma$ as constructed in Appendix 1. 
Given a measure $\mu$ on the underlying measurable space $(M, \mathscr{B}(M))$ described as before, consider for each $n \in \mathbb{N}$ the product measure $\mu^{\otimes n}$ on $\left(M^{n}, \mathscr{B}\left(M^{n}\right)\right)$. Since $\mu^{\otimes n}\left(M^{n} \backslash \widetilde{M^{n}}\right)=0$, one may consider for each $\Lambda \in \mathscr{B}_{c}(M)$ the restriction of $\mu^{\otimes n}$ to $\left(\widetilde{\Lambda^{n}}, \mathscr{B}\left(\widetilde{\Lambda^{n}}\right)\right)$, which is a finite measure, and then the image measure $\mu_{\Lambda}^{(n)}$ on $\left(\Gamma_{\Lambda}^{(n)}, \mathscr{B}\left(\Gamma_{\Lambda}^{(n)}\right)\right)$ under the mapping $\operatorname{sym}_{\Lambda}^{n}$,

$$
\mu_{\Lambda}^{(n)}:=\mu^{\otimes n} \circ\left(\operatorname{sym}_{\Lambda}^{n}\right)^{-1}
$$

For $n=0$ we set $\mu_{\Lambda}^{(0)}:=1$. $^{1}$ Now, for each $0<\alpha<1$ one may define a probability measure $\pi_{\mu, \Lambda}^{\alpha}$ on $\left(\Gamma_{\Lambda}, \mathscr{B}\left(\Gamma_{\Lambda}\right)\right)$ by

$$
\pi_{\mu, \Lambda}^{\alpha}:=\sum_{n=0}^{\infty} \frac{E_{\alpha}^{(n)}(-\mu(\Lambda))}{n !} \mu_{\Lambda}^{(n)}
$$

The family $\left\{\pi_{\mu, \Lambda}^{\alpha}: \Lambda \in \mathscr{B}_{c}(M)\right\}$ of probability measures yields a probability measure on $(\Gamma, \mathscr{B}(\Gamma))$. In fact, this family is consistent, that is,

$$
\pi_{\mu, \Lambda_{1}}^{\alpha}=\pi_{\mu, \Lambda_{2}}^{\alpha} \circ p_{\Lambda_{2}, \Lambda_{1}}^{-1}, \quad \forall \Lambda_{1}, \Lambda_{2} \in \mathscr{B}_{c}(M), \Lambda_{1} \subset \Lambda_{2}
$$

and thus, by the version of Kolmogorov's theorem for the projective limit space $(\Gamma, \mathscr{B}(\Gamma))\left[15\right.$, Chap. V Theorem 5.1], the family $\left\{\pi_{\mu, \Lambda}^{\alpha}: \Lambda \in \mathscr{B}_{c}(M)\right\}$ determines uniquely a measure $\pi_{\mu}^{\alpha}$ on $(\Gamma, \mathscr{B}(\Gamma))$ such that

$$
\pi_{\mu, \Lambda}^{\alpha}=\pi_{\mu}^{\alpha} \circ p_{\Lambda}^{-1}, \quad \forall \Lambda \in \mathscr{B}_{c}(M)
$$

Let us now compute the characteristic functional of the measure $\pi_{\mu} \alpha$. Given a $\varphi \in \mathscr{D}(M)$ we have supp $\varphi \subset \Lambda$ for some $\Lambda \in \mathscr{B}_{c}(M)$, meaning that

$$
\langle\gamma, \varphi\rangle=\left\langle p_{\Lambda}(\gamma), \varphi\right\rangle, \quad \forall \gamma \in \Gamma
$$

Thus

$$
\int_{\Gamma} e^{i\langle\gamma, \varphi\rangle} d \pi_{\mu}^{\alpha}(\gamma)=\int_{\Gamma_{\Lambda}} e^{i\langle\gamma, \varphi\rangle} d \pi_{\mu, \Lambda}^{\alpha}(\gamma)
$$

and the infinite divisibility (11) of the measure $\pi_{\mu, \Lambda}^{\alpha}$ yields for the right-hand side of the equality

$$
\sum_{n=0}^{\infty} \frac{E_{\alpha}^{(n)}(-\mu(\Lambda))}{n !} \int_{\Lambda^{n}} e^{i\left(\varphi\left(x_{1}\right)+\ldots+\varphi\left(x_{n}\right)\right)} d \mu^{\otimes n}(x)=\sum_{n=0}^{\infty} \frac{E_{\alpha}^{(n)}(-\mu(\Lambda))}{n !}\left(\int_{\Lambda} e^{i \varphi(x)} d \mu(x)\right)^{n}
$$

which corresponds to the Taylor expansion of the function

1 Of course this construction holds for any Borel set $Y \in \mathscr{B}(M)$. In this case, $\mu_{Y}^{(n)}\left(\Gamma_{Y}^{(n)}\right)<\infty$ provided $\mu(Y)<\infty$. For more details and proofs see e.g. [16, 17]. 


$$
E_{\alpha}\left(\int_{\Lambda}\left(e^{i \varphi(x)}-1\right) d \mu(x)\right)=E_{\alpha}\left(\int_{M}\left(e^{i \varphi(x)}-1\right) d \mu(x)\right)
$$

In other words, the characteristic functional of the measure $\pi_{\mu}^{\alpha}$ coincides with the characteristic functional of the probability measure given by Theorem 1 through the Bochner-Minlos theorem.

Similarly to the $\alpha=1$ case, this shows that the probability measure on $\left(\mathscr{D}^{\prime}(M), \mathscr{C}_{\sigma}\left(\mathscr{D}^{\prime}(M)\right)\right)$ given by Theorem 1 is actually supported on generalized functions of the form $\sum_{x \in \gamma} \delta_{x}, \gamma \in \Gamma$. Thus, each fractional Poisson measure $\pi_{\mu}^{\alpha}$ can either be consider on $(\Gamma, \mathscr{B}(\Gamma))$ or on $\left(\mathscr{D}^{\prime}, \mathscr{C}_{\sigma}\left(\mathscr{D}^{\prime}(M)\right)\right)$ where, in contrast to $\Gamma, \mathscr{D}^{\prime}(M) \supset \Gamma$ is a linear space. Since $\pi_{\mu}^{\alpha}(\Gamma)=1$, the measure space $\left(\mathscr{D}^{\prime}(M), \mathscr{C}_{\sigma}\left(\mathscr{D}^{\prime}(M)\right), \pi_{\mu}^{\alpha}\right)$ can, in this way, be regarded as a linear extension of the fractional Poisson space $\left(\Gamma, \mathscr{B}(\Gamma), \pi_{\mu}^{\alpha}\right)$.

\subsection{Fractional Poisson Analysis}

\subsubsection{Fractional Lebesgue-Poisson Measure and Unitary Isomorphisms}

Let us now consider the space of finite configurations

$$
\Gamma_{0}:=\bigsqcup_{n=0}^{\infty} \Gamma_{M}^{(n)}
$$

endowed with the topology of disjoint union of topological spaces, with the corresponding Borel $\sigma$-algebra $\mathscr{B}\left(\Gamma_{0}\right)$ and the so-called $K$-transform [16, 18-22], a mapping which maps functions defined on $\Gamma_{0}$ into functions defined on $\Gamma$. By definition, given a $\mathscr{B}\left(\Gamma_{0}\right)$-measurable function $G$ with local support, that is, $G\left\lceil\Gamma_{0} \backslash \Gamma_{\Lambda} \equiv 0\right.$ for some $\Lambda \in \mathscr{B}_{c}(M)$, the $K$-transform of $G$ is a mapping $K G: \Gamma \rightarrow \mathbb{R}$ defined at each $\gamma \in \Gamma$ by

$$
(K G)(\gamma):=\sum_{\substack{\eta \subset \gamma \\|\eta|<\infty}} G(\eta)
$$

Note that for every such function $G$ the sum in (12) has only a finite number of summands different from zero, and thus $K G$ is a well-defined function on $\Gamma$. Moreover, if $G$ has support described as before, then the restriction $(K G) \uparrow_{\Gamma_{\Lambda}}$ is a $\mathscr{B}\left(\Gamma_{\Lambda}\right)$ measurable function and $(K G)(\gamma)=(K G)\left\lceil_{\Gamma_{\Lambda}}\left(\gamma_{\Lambda}\right)\right.$ for all $\gamma \in \Gamma$.

In terms of the dual operator $K^{*}$ of the $K$-transform, this means that the image of a probability measure on $\Gamma$ under $K^{*}$ yields a measure on $\Gamma_{0}$. More precisely, given a probability measure $v$ on $(\Gamma, \mathscr{B}(\Gamma))$ with finite local moments of all orders, that is,

$$
\int_{\Gamma}\left|\gamma_{\Lambda}\right|^{n} d \nu(\gamma)<\infty \text { for all } n \in \mathbb{N} \text { and all } \Lambda \in \mathscr{B}_{c}(M)
$$


then $K^{*} v$ is a measure on $\left(\Gamma_{0}, \mathscr{B}\left(\Gamma_{0}\right)\right)$ defined on each bounded $\mathscr{B}\left(\Gamma_{0}\right)$-measurable set $A$ by

$$
\left(K^{*} \nu\right)(A)=\int_{\Gamma}\left(K 1_{A}\right)(\gamma) d v(\gamma)
$$

The measure $K^{*} v$ is called the correlation measure corresponding to $v$. In particular, for the Poisson measure $\pi_{\mu}$, the correlation measure corresponding to $\pi_{\mu}$ is called the Lebesgue-Poisson measure

$$
\lambda_{\mu}:=\sum_{n=0}^{\infty} \frac{1}{n !} \mu^{(n)}, \quad \mu^{(n)}:=\mu^{\otimes n} \circ\left(\operatorname{sym}_{M}^{n}\right)^{-1}
$$

For more details and proofs see e.g. [16].

Theorem 2 For each $0<\alpha<1$, the correlation measure corresponding to the fractional Poisson measure $\pi_{\mu}^{\alpha}$ is the measure on $\left(\Gamma_{0}, \mathscr{B}\left(\Gamma_{0}\right)\right)$ given by

$$
\lambda_{\mu}^{\alpha}:=\sum_{n=0}^{\infty} \frac{1}{\Gamma(\alpha n+1)} \mu^{(n)}
$$

In other words, $d \lambda_{\mu}^{\alpha}=E_{\alpha}^{(|\cdot|)}(0) d \lambda_{\mu}$.

In the sequel we call the measure $\lambda_{\mu}^{\alpha}$ the fractional Lebesgue-Poisson measure.

Proof Let $A$ be a bounded $\mathscr{B}\left(\Gamma_{0}\right)$-measurable set, that is,

$$
A \subset \bigsqcup_{n=0}^{N} \Gamma_{\Lambda}^{(n)}
$$

for some $N \in \mathbb{N}_{0}$ and some $\Lambda \in \mathscr{B}_{c}(M)$. By the previous considerations, this means that for all $\gamma \in \Gamma$ one has $\left(K 1_{A}\right)(\gamma)=\left(K 1_{A}\right)\left(\gamma_{\Lambda}\right)$, and thus

$$
\begin{aligned}
\int_{\Gamma}\left(K 1_{A}\right)(\gamma) d \pi_{\mu}^{\alpha}(\gamma) & =\int_{\Gamma_{\Lambda}}\left(K 1_{A}\right)(\gamma) d \pi_{\mu, \Lambda}^{\alpha}(\gamma) \\
& =\sum_{n=0}^{\infty} \frac{E_{\alpha}^{(n)}(-\mu(\Lambda))}{n !} \int_{\Gamma_{\Lambda}^{(n)}}\left(K 1_{A}\right)(\eta) d \mu_{\Lambda}^{(n)}(\eta) \\
& =\int_{\Gamma_{\Lambda}} E_{\alpha}^{(|\eta|)}(-\mu(\Lambda))\left(K 1_{A}\right)(\eta) d \lambda_{\mu}(\eta)
\end{aligned}
$$

Observe that the latter integral is with respect to the Lebesgue-Poisson measure $\lambda_{\mu}$, which properties are well-known (see e.g. [16]). In particular, those yield 


$$
\begin{aligned}
& \int_{\Gamma_{\Lambda}} E_{\alpha}^{(|\eta|)}(-\mu(\Lambda))\left(K 1_{A}\right)(\eta) d \lambda_{\mu}(\eta) \\
= & \int_{\Gamma_{0}} E_{\alpha}^{(|\eta|)}(-\mu(\Lambda)) \sum_{\xi \subset \eta} 1_{A}(\xi) 11_{\Gamma_{\Lambda}}(\eta \backslash \xi) d \lambda_{\mu}(\eta) \\
= & \int_{\Gamma_{0}} 1_{A}(\eta)\left(\int_{\Gamma_{0}} E_{\alpha}^{(|\eta \cup \xi|)}(-\mu(\Lambda)) 1_{\Gamma_{\Lambda}}(\xi) d \lambda_{\mu}(\xi)\right) d \lambda_{\mu}(\eta)
\end{aligned}
$$

where for each $\eta \in \Gamma_{0}$ fixed, i.e., $\eta \in \Gamma_{M}^{(m)}$ for some $m \in \mathbb{N}_{0}$, the integral between brackets is given by

$$
\begin{aligned}
& \sum_{n=0}^{\infty} \frac{1}{n !} \int_{\Gamma_{M}^{(n)}} E_{\alpha}^{(|\eta \cup \xi|)}(-\mu(\Lambda)) 1_{\Gamma_{\Lambda}}(\xi) d \mu^{(n)}(\xi) \\
= & \sum_{n=0}^{\infty} \frac{E_{\alpha}^{(m+n)}(-\mu(\Lambda))}{n !}(\mu(\Lambda))^{n} \\
= & E_{\alpha}^{(m)}(-\mu(\Lambda)+\mu(\Lambda))
\end{aligned}
$$

As a result,

$$
\int_{\Gamma}\left(K 1_{A}\right)(\gamma) d \pi_{\mu}^{\alpha}(\gamma)=\int_{\Gamma_{0}} 1_{A}(\eta) E_{\alpha}^{(|\eta|)}(0) d \lambda_{\mu}(\eta)
$$

showing that the correlation measure corresponding to $\pi_{\mu}^{\alpha}$ is absolutely continuous with respect to the Lebesgue-Poisson measure $\lambda_{\mu}$. Moreover, denoting such a correlation measure by $\lambda_{\mu}^{\alpha}$, the density is given by $\frac{d \lambda_{\mu}^{\alpha}}{d \lambda_{\mu}}=E_{\alpha}^{(|\cdot|)}(0)$.

To conclude, notice that for each $n \in \mathbb{N}_{0}$ one has

$$
E_{\alpha}^{(n)}(0)=\frac{n !}{\Gamma(\alpha n+1)}
$$

which combined with the definition of the measure $\lambda_{\mu}$ leads to (13).

Throughout this work all $L^{p}$-spaces consist of complex-valued functions. For simplicity, the $L^{p}$-spaces with respect to a measure $v$ will be shortly denoted by $L^{p}(v)$ if the underlying measurable space is clear from the context.

Corollary 1 We have $G \in L^{2}\left(\lambda_{\mu}^{\alpha}\right)$ if and only if $G \sqrt{E_{\alpha}^{(|\cdot|)}(0)} \in L^{2}\left(\lambda_{\mu}\right)$. Then,

$$
\|G\|_{L^{2}\left(\lambda_{\mu}^{\alpha}\right)}=\left\|G \sqrt{E_{\alpha}^{(|\cdot|)}(0)}\right\|_{L^{2}\left(\lambda_{\mu}\right)}
$$

This result states that there is a unitary isomorphism between the spaces $L^{2}\left(\lambda_{\mu}^{\alpha}\right)$ and $L^{2}\left(\lambda_{\mu}\right)$ : 


$$
\begin{aligned}
I_{\alpha}: L^{2}\left(\lambda_{\mu}^{\alpha}\right) & \rightarrow L^{2}\left(\lambda_{\mu}\right) \\
I_{\alpha}(G) & :=G \sqrt{E_{\alpha}^{(|\cdot|)}(0)}
\end{aligned}
$$

Hence, through $I_{\alpha}$ one may extend the unitary isomorphisms defined between the space $L^{2}\left(\lambda_{\mu}\right)$ and the (Bose or symmetric) Fock space $\operatorname{Exp} L^{2}(\mu)$ and between the space $L^{2}\left(\lambda_{\mu}\right)$ and $L^{2}\left(\pi_{\mu}\right)[17]$ to $L^{2}\left(\lambda_{\mu}^{\alpha}\right), 0<\alpha \leq 1$ :

$$
\begin{aligned}
L^{2}\left(\lambda_{\mu}^{\alpha}\right) \stackrel{I_{\alpha}}{\mapsto} L^{2}\left(\lambda_{\mu}\right) & \stackrel{I_{\lambda \pi}}{\mapsto} \quad L^{2}\left(\pi_{\mu}\right) \quad \stackrel{I_{\pi}}{\mapsto} \operatorname{Exp}^{2}(\mu) \\
G \quad \mapsto G \sqrt{E_{\alpha}^{(\cdot \mid \cdot)}(0)} & \mapsto \sum_{n=0}^{\infty}\left\langle C_{n}^{\mu}, g^{(n)}\right\rangle \mapsto\left(g^{(n)}\right)_{n=0}^{\infty}
\end{aligned}
$$

for

$$
g^{(n)}\left(x_{1}, \ldots, x_{n}\right):=\frac{\sqrt{E_{\alpha}^{(n)}(0)}}{n !} G\left(\left\{x_{1}, \ldots, x_{n}\right\}\right), \quad g^{(0)}:=E_{\alpha}(0) G(\emptyset)=G(\emptyset)
$$

and $C_{n}^{\mu}$ a Charlier kernel.

In particular, the image of a Fock coherent state $e(f):=\left(\frac{f^{\otimes n}}{n !}\right)_{n=0}^{\infty}, f \in L^{2}(\mu)$, under $\left(I_{\pi} \circ I_{\lambda \pi}\right)^{-1}$ is the (Lebesgue-Poisson) coherent state $e_{\lambda}(f): \Gamma_{0} \rightarrow \mathbb{C}$ defined for any $\mathscr{B}(M)$-measurable function $f: M \rightarrow \mathbb{C}$ by

$$
e_{\lambda}(f, \eta):=\prod_{x \in \eta} f(x), \eta \in \Gamma_{0} \backslash\{\emptyset\}, \quad e_{\lambda}(f, \emptyset):=1
$$

This definition implies that $e_{\lambda}(f) \in L^{p}\left(\lambda_{\mu}\right)$ whenever $f \in L^{p}(\mu)$ for some $p \geq 1$. Moreover, $\left\|e_{\lambda}(f)\right\|_{L^{p}\left(\lambda_{\mu}\right)}^{p}=\exp \left(\|f\|_{L^{p}(\mu)}^{p}\right)$. For $\alpha \neq 1$, the following result holds.

Proposition 1 Let $0<\alpha<1$ and $p \geq 1$ be given. For all $f \in L^{p}(\mu)$ we have $e_{\lambda}(f) \in L^{p}\left(\lambda_{\mu}^{\alpha}\right)$ and

$$
\left\|e_{\lambda}(f)\right\|_{L^{p}\left(\lambda_{\mu}^{\alpha}\right)}^{p}=E_{\alpha}\left(\|f\|_{L^{p}(\mu)}^{p}\right)
$$

Proof By Theorem 2, given a $f \in L^{p}(\mu)$ for some $p \geq 1$,

$$
\begin{aligned}
\left\|e_{\lambda}(f)\right\|_{L^{p}\left(\lambda_{\mu}^{\alpha}\right)}^{p} & =\int_{\Gamma_{0}}\left|e_{\lambda}(f, \eta)\right|^{p} E_{\alpha}^{(|\eta|)}(0) d \lambda_{\mu}(\eta) \\
& =\sum_{n=0}^{\infty} \frac{1}{\Gamma(\alpha n+1)}\left(\int_{M}|f(x)|^{p} d \mu(x)\right)^{n}
\end{aligned}
$$

which by the Taylor expansion (7) is equal to $E_{\alpha}\left(\int_{M}|f(x)|^{p} d \mu(x)\right)$.

According to the latter considerations, the realization of a coherent state $e(f)$, $f \in L^{2}(\mu)$, in a $L^{2}\left(\lambda_{\mu}^{\alpha}\right)$ space is $\lambda_{\mu}$-a.e. given by 


$$
I_{\alpha}^{-1} e_{\lambda}(f)=\frac{e_{\lambda}(f)}{\sqrt{E_{\alpha}^{(|\cdot|)}(0)}}
$$

In addition, given a dense subspace $L \subseteq L^{2}(\mu)$, the set $\left\{I_{\alpha}^{-1} e_{\lambda}(f): f \in L\right\}$ is total in $L^{2}\left(\lambda_{\mu}^{\alpha}\right)$. As in the Lebesgue-Poisson case, we define the fractional (LebesguePoisson) coherent state $e_{\alpha}(f): \Gamma_{0} \rightarrow \mathbb{C}$ corresponding to a $\mathscr{B}(M)$-measurable function $f$ by

$$
e_{\alpha}(f, \eta):=\frac{e_{\lambda}(f, \eta)}{\sqrt{E_{\alpha}^{(|\eta|)}(0)}}, \quad \forall \eta \in \Gamma_{0}
$$

\subsubsection{Annihilation and Creation Operators}

The unitary isomorphism between the Fock space and $L^{2}\left(\lambda_{\mu}\right)$ provides natural operators on the space $L^{2}\left(\lambda_{\mu}\right)$ by carrying over the standard Fock space operators. In particular, the annihilation and the creation operators, for which the images in $L^{2}\left(\lambda_{\mu}\right)$ are well-known, see e.g. [23],

$$
\left(a_{\lambda}^{-}(\varphi) G\right)(\eta):=\int_{M} G(\eta \cup\{x\}) \varphi(x) d \mu(x), \quad \eta \in \Gamma_{0}
$$

and

$$
\left(a_{\lambda}^{+}(\varphi) G\right)(\eta):=\sum_{x \in \eta} G(\eta \backslash\{x\}) \varphi(x), \quad \lambda_{\mu}-a . a . \eta \in \Gamma_{0}
$$

Here $\varphi \in \mathscr{D}(M)$ and $G$ is a complex-valued bounded $\mathscr{B}\left(\Gamma_{0}\right)$-measurable function with bounded support, i.e., $\left.G\right|_{\Gamma_{0} \backslash\left(\bigsqcup_{n=0}^{N} \Gamma_{\Lambda}^{(n)}\right)} \equiv 0$ for some $\Lambda \in \mathscr{B}_{c}(M)$ and some $N \in \mathbb{N}_{0}$. In the sequel we denote the space of such functions $G$ by $B_{b s}\left(\Gamma_{0}\right)$.

For more details and proofs see e.g. [17, 24] and the references therein.

Through the unitary isomorphism $I_{\alpha}^{-1}, 0<\alpha<1$, the same Fock space operators can naturally be carried over to the space $L^{2}\left(\lambda_{\mu}^{\alpha}\right)$.

Proposition 2 For each $\varphi \in \mathscr{D}(M)$, the following relations hold on $B_{b s}\left(\Gamma_{0}\right)$ :

$$
a_{\alpha}^{-}(\varphi):=I_{\alpha}^{-1} a_{\lambda}^{-}(\varphi) I_{\alpha}=\sqrt{\frac{E_{\alpha}^{(|\cdot|+1)}(0)}{E_{\alpha}^{(|\cdot|)}(0)}} a_{\lambda}^{-}(\varphi)
$$

and

$$
a_{\alpha}^{+}(\varphi):=I_{\alpha}^{-1} a_{\lambda}^{+}(\varphi) I_{\alpha}=\sqrt{\frac{E_{\alpha}^{(|\cdot|-1)}(0)}{E_{\alpha}^{(|\cdot|)}(0)}} a_{\lambda}^{+}(\varphi)
$$


Proof One first observes that $I_{\alpha}$ maps the space $B_{b s}\left(\Gamma_{0}\right)$ into itself. In fact, given a $G \in B_{b s}\left(\Gamma_{0}\right)$, i.e., $\left.G\right|_{\Gamma_{0} \backslash\left(\bigsqcup_{n=0}^{N} \Gamma_{\Lambda}^{(n)}\right)} \equiv 0$ for some $\Lambda \in \mathscr{B}_{c}(M)$ and some $N \in \mathbb{N}_{0}$, one has

$$
\left|\left(I_{\alpha} G\right)(\eta)\right|=\sqrt{E_{\alpha}^{(|\eta|)}(0)}|G(\eta)| \leq \max _{0 \leq n \leq N} \sqrt{\frac{n !}{\Gamma(\alpha n+1)}} \sup _{\eta \in \Gamma_{0}}(|G(\eta)|), \quad \forall \eta \in \Gamma_{0}
$$

showing that $I_{\alpha} G$ is bounded. Since the support of $I_{\alpha} G$ clearly coincides with the support of $G$, this means that $I_{\alpha} G \in B_{b s}\left(\Gamma_{0}\right)$.

Hence, given a $G \in B_{b s}\left(\Gamma_{0}\right)$, for all $\eta \in \Gamma_{0}$ one has

$$
\begin{aligned}
\left(a_{\lambda}^{-}(\varphi)\left(I_{\alpha} G\right)\right)(\eta) & =\int_{M}\left(I_{\alpha} G\right)(\eta \cup\{x\}) \varphi(x) d \mu(x) \\
& =\sqrt{E_{\alpha}^{(|\eta|+1)}(0)}\left(a_{\lambda}^{-}(\varphi) G\right)(\eta)
\end{aligned}
$$

which proves the first equality by calculating the image of both sides under $I_{\alpha}^{-1}$. A similar procedure applied to $a_{\alpha}^{+}(\varphi)$ completes the proof.

\subsubsection{Second Quantization Operators}

Given a contraction operator $B$ on $L^{2}(\mu)$ one may define a contraction operator $\operatorname{Exp} B$ on the Fock space $\operatorname{Exp} L^{2}(\mu)$ acting on coherent states $e(f), f \in L^{2}(\mu)$, by $\operatorname{Exp} B(e(f))=e(B f)$. In particular, given a positive self-adjoint operator $A$ on $L^{2}(\mu)$ and the contraction semigroup $e^{-t A}, t \geq 0$, one can define a contraction semigroup $\operatorname{Exp}\left(e^{-t A}\right)$ on $\operatorname{Exp} L^{2}(\mu)$ in this way. The generator is the well-known second quantization operator corresponding to $A$. We denote it by $d \operatorname{Exp} A$. Through the unitary isomorphism between the Fock space and the space $L^{2}\left(\lambda_{\mu}\right)$ one may then define the corresponding operator in $L^{2}\left(\lambda_{\mu}\right)$. We denote the (Lebesgue-Poisson) second quantization operator corresponding to $A$ by $H_{A}^{L P}$. The action of $H_{A}^{L P}$ on coherent states is given by

$$
\left(H_{A}^{L P} e_{\lambda}(f)\right)(\eta)=\sum_{x \in \eta}(A f)(x) e_{\lambda}(f, \eta \backslash\{x\}), \quad f \in D(A)
$$

Through the unitary isomorphism $I_{\alpha}^{-1}, 0<\alpha<1$, the second quantization operator can also be carried over to the space $L^{2}\left(\lambda_{\mu}^{\alpha}\right)$ :

$$
H_{A}^{\alpha}:=I_{\alpha}^{-1} H_{A}^{L P} I_{\alpha}
$$

Proposition 3 For any $f \in D(A)$ we have 


$$
\left(H_{A}^{\alpha} e_{\alpha}(f)\right)(\eta)=\sqrt{\frac{E_{\alpha}^{(|\eta|-1)}(0)}{E_{\alpha}^{(|\eta|)}(0)}} \sum_{x \in \eta}(A f)(x) e_{\alpha}(f, \eta \backslash\{x\})
$$

Proof According to (14), $I_{\alpha} e_{\alpha}(f)=e_{\lambda}(f)$, and thus for $\lambda_{\mu}$-a.a. $\eta \in \Gamma_{0}$,

$$
\left(H_{A}^{L P}\left(I_{\alpha} e_{\alpha}(f)\right)\right)(\eta)=\left(H_{A}^{L P} e_{\lambda}(f)\right)(\eta)=\sqrt{E_{\alpha}^{(|\eta|-1)}(0)} \sum_{x \in \eta}(A f)(x) e_{\alpha}(f, \eta \backslash\{x\})
$$

leading to the required result by calculating the image of both sides under $I_{\alpha}^{-1}$.

\section{Appendix 1: The Infinite-Dimensional Poisson Measure and Configuration Spaces}

Here a short summary is given of the properties of the infinite-dimensional Poisson measure and its support on configuration spaces [12, 13, 16-19, 24].

An infinite-dimensional generalization of the characteristic function of the Poisson measure is obtained by generalizing (5) to

$$
C(\varphi)=e^{\int\left(e^{i \varphi(x)}-1\right) d \mu(x)}
$$

for test functions $\varphi \in \mathscr{D}(M)$ in the space of $C^{\infty}$-functions of compact support in a manifold $M$. It is easy to prove, using the Bochner-Minlos theorem, that $C$ is indeed the Fourier transform of a measure on the distribution space $\mathscr{D}^{\prime}(M)$.

A support for this measure is obtained in the configuration space $\Gamma:=\Gamma_{M}$ over the manifold $M$, defined as the set of all locally finite subsets of $M$ (simple configurations)

$$
\Gamma:=\{\gamma \subset M:|\gamma \cap K|<\infty \text { for any compact } K \subset M\}
$$

Here $|A|$ denotes the cardinality of the set $A$. As usual one identifies each $\gamma \in \Gamma$ with a non-negative integer-valued Radon measure,

$$
\Gamma \ni \gamma \mapsto \sum_{x \in \gamma} \delta_{x} \in \mathscr{M}(M)
$$

where $\delta_{x}$ is the Dirac measure with unit mass at $x$ and $\mathscr{M}(M)$ denotes the set of all non-negative Radon measures on $M$. In this way the space $\Gamma$ can be endowed with the relative topology as a subset of the space $\mathscr{M}(M)$ with the vague topology, i.e., the weakest topology on $\Gamma$ for which the mappings 


$$
\Gamma \ni \gamma \mapsto\langle\gamma, f\rangle:=\int_{M} f(x) d \gamma(x)=\sum_{x \in \gamma} f(x)
$$

are continuous for all real-valued continuous functions $f$ on $M$ with compact support. Denote the corresponding Borel $\sigma$-algebra on $\Gamma$ by $\mathscr{B}(\Gamma)$.

For each $Y \in \mathscr{B}(M)$ let us consider the space $\Gamma_{Y}$ of all configurations contained in $Y, \Gamma_{Y}:=\{\gamma \in \Gamma:|\gamma \cap(X \backslash Y)|=0\}$, and the space $\Gamma_{Y}^{(n)}$ of $n$-point configurations,

$$
\Gamma_{Y}^{(n)}:=\left\{\gamma \in \Gamma_{Y}:|\gamma|=n\right\}, n \in \mathbb{N}, \quad \Gamma_{Y}^{(0)}:=\{\emptyset\}
$$

A topological structure may be introduced on $\Gamma_{Y}^{(n)}$ through the natural surjective mapping of $\widetilde{Y^{n}}:=\left\{\left(x_{1}, \ldots, x_{n}\right): x_{i} \in Y, x_{i} \neq x_{j}\right.$ if $\left.i \neq j\right\}$ onto $\Gamma_{Y}^{(n)}$,

$$
\begin{aligned}
\operatorname{sym}_{Y}^{n}: \widetilde{Y^{n}} & \longrightarrow \Gamma_{Y}^{(n)} \\
\left(x_{1}, \ldots, x_{n}\right) \longmapsto & \left.\longmapsto x_{1}, \ldots, x_{n}\right\}
\end{aligned}
$$

which is at the origin of a bijection between $\Gamma_{Y}^{(n)}$ and the symmetrization $\widetilde{Y^{n}} / S_{n}$ of $\widetilde{Y^{n}}, S_{n}$ being the permutation group over $\{1, \ldots, n\}$. Thus, $\operatorname{sym}_{Y}^{n}$ induces a metric on $\Gamma_{Y}^{(n)}$ and the corresponding Borel $\sigma$-algebra $\mathscr{B}\left(\Gamma_{Y}^{(n)}\right)$ on $\Gamma_{Y}^{(n)}$.

For $\Lambda \in \mathscr{B}(M)$ with compact closure $\left(\Lambda \in \mathscr{B}_{c}(M)\right)$, it clearly follows from (16) that

$$
\Gamma_{\Lambda}=\bigsqcup_{n=0}^{\infty} \Gamma_{\Lambda}^{(n)}
$$

the $\sigma$-algebra $\mathscr{B}\left(\Gamma_{\Lambda}\right)$ being defined by the disjoint union of the $\sigma$-algebras $\mathscr{B}\left(\Gamma_{Y}^{(n)}\right)$, $n \in \mathbb{N}_{0}$.

For each $\Lambda \in \mathscr{B}_{c}(M)$ there is a natural measurable mapping $p_{\Lambda}: \Gamma \rightarrow \Gamma_{\Lambda}$. Similarly, given any pair $\Lambda_{1}, \Lambda_{2} \in \mathscr{B}_{c}(M)$ with $\Lambda_{1} \subset \Lambda_{2}$ there is a natural mapping $p_{\Lambda_{2}, \Lambda_{1}}: \Gamma_{\Lambda_{2}} \rightarrow \Gamma_{\Lambda_{1}}$. They are defined, respectively, by

$$
\begin{aligned}
p_{\Lambda}: \Gamma \longrightarrow \Gamma_{\Lambda} & p_{\Lambda_{2}, \Lambda_{1}}: \Gamma_{\Lambda_{2}} \longrightarrow \Gamma_{\Lambda_{1}} \\
\gamma & \longmapsto \gamma_{\Lambda}:=\gamma \cap \Lambda
\end{aligned}
$$

It can be shown that $(\Gamma, \mathscr{B}(\Gamma))$ coincides (up to an isomorphism) with the projective limit of the measurable spaces $\left(\Gamma_{\Lambda}, \mathscr{B}\left(\Gamma_{\Lambda}\right)\right), \Lambda \in \mathscr{B}_{c}(M)$, with respect to the projection $p_{\Lambda}$, i.e., $\mathscr{B}(\Gamma)$ is the smallest $\sigma$-algebra on $\Gamma$ with respect to which all projections $p_{\Lambda}, \Lambda \in \mathscr{B}_{c}(M)$, are measurable.

Let now $\mu$ be a measure on the underlying measurable space $(M, \mathscr{B}(M))$ and consider for each $n \in \mathbb{N}$ the product measure $\mu^{\otimes n}$ on $\left(M^{n}, \mathscr{B}\left(M^{n}\right)\right)$. Since $\mu^{\otimes n}\left(M^{n} \backslash \widetilde{M^{n}}\right)=0$, one may consider for each $\Lambda \in \mathscr{B}_{c}(M)$ the restriction of $\mu^{\otimes n}$ to $\left(\widetilde{\Lambda^{n}}, \mathscr{B}\left(\widetilde{\Lambda^{n}}\right)\right)$, which is a finite measure, and then the image measure $\mu_{\Lambda}^{(n)}$ on $\left(\Gamma_{\Lambda}^{(n)}, \mathscr{B}\left(\Gamma_{\Lambda}^{(n)}\right)\right)$ under the mapping $\operatorname{sym}_{\Lambda}^{n}$, 


$$
\mu_{\Lambda}^{(n)}:=\mu^{\otimes n} \circ\left(\operatorname{sym}_{\Lambda}^{n}\right)^{-1}
$$

For $n=0$ we set $\mu_{\Lambda}^{(0)}:=1$. Now, one may define a probability measure $\pi_{\mu, \Lambda}$ on $\left(\Gamma_{\Lambda}, \mathscr{B}\left(\Gamma_{\Lambda}\right)\right)$ by

$$
\pi_{\mu, \Lambda}:=\sum_{n=0}^{\infty} \frac{\exp (-\mu(\Lambda))}{n !} \mu_{\Lambda}^{(n)}
$$

The family $\left\{\pi_{\mu, \Lambda}: \Lambda \in \mathscr{B}_{c}(M)\right\}$ of probability measures yields a probability measure on $(\Gamma, \mathscr{B}(\Gamma))$ with the $\pi_{\mu, \Lambda}$ as projections. This family is consistent, that is,

$$
\pi_{\mu, \Lambda_{1}}=\pi_{\mu, \Lambda_{2}} \circ p_{\Lambda_{2}, \Lambda_{1}}^{-1}, \quad \forall \Lambda_{1}, \Lambda_{2} \in \mathscr{B}_{c}(M), \Lambda_{1} \subset \Lambda_{2}
$$

and thus, by the version of Kolmogorov's theorem for the projective limit space $(\Gamma, \mathscr{B}(\Gamma))$, the family $\left\{\pi_{\mu, \Lambda}: \Lambda \in \mathscr{B}_{c}(M)\right\}$ determines uniquely a measure $\pi_{\mu}$ on $(\Gamma, \mathscr{B}(\Gamma))$ such that

$$
\pi_{\mu, \Lambda}=\pi_{\mu} \circ p_{\Lambda}^{-1}, \quad \forall \Lambda \in \mathscr{B}_{c}(M)
$$

The next step is to compute the characteristic functional of the measure $\pi_{\mu}$. Given a $\varphi \in \mathscr{D}(M)$ we have $\operatorname{supp} \varphi \subset \Lambda$ for some $\Lambda \in \mathscr{B}_{c}(M)$, meaning that

$$
\langle\gamma, \varphi\rangle=\left\langle p_{\Lambda}(\gamma), \varphi\right\rangle, \quad \forall \gamma \in \Gamma
$$

Thus

$$
\int_{\Gamma} e^{i\langle\gamma, \varphi\rangle} d \pi_{\mu}(\gamma)=\int_{\Gamma_{\Lambda}} e^{i\langle\gamma, \varphi\rangle} d \pi_{\mu, \Lambda}(\gamma)
$$

and the definition (17) of the measure $\pi_{\mu, \Lambda}$ yields for the right-hand side of the equality

$$
\sum_{n=0}^{\infty} \frac{\exp (-\mu(\Lambda))}{n !} \int_{\Lambda^{n}} e^{i\left(\varphi\left(x_{1}\right)+\cdots+\varphi\left(x_{n}\right)\right)} d \mu^{\otimes n}(x)=\sum_{n=0}^{\infty} \frac{\exp (-\mu(\Lambda))}{n !}\left(\int_{\Lambda} e^{i \varphi(x)} d \mu(x)\right)^{n}
$$

which corresponds to the Taylor expansion of the characteristic function (15) of the infinite-dimensional Poisson measure

$$
\exp \left(\int_{\Lambda}\left(e^{i \varphi(x)}-1\right) d \mu(x)\right)
$$

This shows that the probability measure on $\left(\mathscr{D}^{\prime}(M), \mathscr{C}_{\sigma}\left(\mathscr{D}^{\prime}(M)\right)\right)$ given by (15) is actually supported on generalized functions of the form $\sum_{x \in \gamma} \delta_{x}, \gamma \in \Gamma$. Thus, the infinite-dimensional Poisson measure $\pi_{\mu}$ can either be considered as a measure on $(\Gamma, \mathscr{B}(\Gamma))$ or on $\left(\mathscr{D}^{\prime}, \mathscr{C}_{\sigma}\left(\mathscr{D}^{\prime}(M)\right)\right)$. Notice that, in contrast to $\Gamma, \mathscr{D}^{\prime}(M) \supset \Gamma$ is a 
linear space. Since $\pi_{\mu}(\Gamma)=1$, the measure space $\left(\mathscr{D}^{\prime}(M), \mathscr{C}_{\sigma}\left(\mathscr{D}^{\prime}(M)\right), \pi_{\mu}\right)$ can, in this way, be regarded as a linear extension of the Poisson space $\left(\Gamma, \mathscr{B}(\Gamma), \pi_{\mu}\right)$.

\section{Appendix 2. Complete Monotonicity of the Mittag-Leffler Function for Complex Arguments}

A positive $C^{\infty}$-function $f$ is said to be completely monotone if for each $k \in \mathbb{N}_{0}$

$$
(-1)^{k} f^{(k)}(t) \geq 0, \quad \forall t>0
$$

According to Bernstein's theorem (see e.g. [25, Chap. XIII.4 Theorem 1]), for functions $f$ such that $f\left(0^{+}\right)=1$ the complete monotonicity property is equivalent to the existence of a probability measure $v$ on $\mathbb{R}_{0}^{+}$such that

$$
f(t)=\int_{0}^{\infty} e^{-t \tau} d \nu(\tau)<\infty, \quad \forall t>0
$$

Pollard in [26] proved the complete monotonicity of $E_{\alpha}, 0<\alpha<1$, for non-positive real arguments showing that

$$
E_{\alpha}(-t)=\int_{0}^{\infty} e^{-t \tau} d v_{\alpha}(\tau), \quad \forall t \geq 0
$$

for $v_{\alpha}$ being the probability measure on $\mathbb{R}_{0}^{+}$

$$
d \nu_{\alpha}(\tau):=\alpha^{-1} \tau^{-1-1 / \alpha} f_{\alpha}\left(\tau^{-1 / \alpha}\right) d \tau
$$

where $f_{\alpha}$ is the $\alpha$-stable probability density given by

$$
\int_{0}^{\infty} e^{-t \tau} f_{\alpha}(\tau) d \tau=e^{-t^{\alpha}}, \quad 0<\alpha<1
$$

The complete monotonicity property and the integral representation (18) of $E_{\alpha}$ may be extended to complex arguments.

Lemma 1 For any $z \in \mathbb{C}$ such that $\operatorname{Re}(z) \geq 0$, the following representation holds

$$
E_{\alpha}(-z)=\int_{0}^{\infty} e^{-z \tau} d v_{\alpha}(\tau), \quad 0<\alpha \leq 1
$$

Proof According to [26], for each $0<\alpha<1$ fixed, for all $t \geq 0$ one has 


$$
\begin{aligned}
E_{\alpha}(-t) & =\int_{0}^{\infty} e^{-t \tau} d \nu_{\alpha}(\tau) \\
& =\sum_{n=0}^{\infty} \frac{(-t)^{n}}{n !} \int_{0}^{\infty} \tau^{n} d \nu_{\alpha}(\tau)
\end{aligned}
$$

Comparing (20) with the Taylor expansion (7) of $E_{\alpha}$, one concludes that the moments of the measure $v_{\alpha}$ are given by

$$
m_{n}\left(v_{\alpha}\right):=\int_{0}^{\infty} \tau^{n} d v_{\alpha}(\tau)=\frac{n !}{\Gamma(\alpha n+1)}, \quad n \in \mathbb{N}_{0}
$$

For complex values $z$ let

$$
I(-z):=\int_{0}^{\infty} e^{-z \tau} d v_{\alpha}(\tau)
$$

which is finite provided $\operatorname{Re}(z) \geq 0$. For each $z \in \mathbb{C}$ such that $\operatorname{Re}(z) \geq 0$ one then obtains

$$
I(-z)=\sum_{n=0}^{\infty} \frac{(-z)^{n}}{n !}\left(\int_{0}^{\infty} \tau^{n} d v_{\alpha}(\tau)\right)=\sum_{n=0}^{\infty} \frac{(-z)^{n}}{n !} m_{n}\left(v_{\alpha}\right)=\sum_{n=0}^{\infty} \frac{(-z)^{n}}{\Gamma(\alpha n+1)}=E_{\alpha}(-z)
$$

leading to the integral representation

$$
E_{\alpha}(-z)=\int_{0}^{\infty} e^{-z \tau} d v_{\alpha}(\tau)
$$

for all $z \in \mathbb{C}$ such that $\operatorname{Re}(z) \geq 0$.

\section{References}

1. Goldin, G.A.: Nonrelativistic current algebras as unitary representations of groups. J. Math. Phys. 12, 462-487 (1971)

2. Goldin, G.A., Grodnik, J., Powers, R.T., Sharp, D.H.: Nonrelativistic current algebra in the N/V limit. J. Math. Phys. 15, 88-100 (1974)

3. Goldin, G.A.: Lectures on diffeomorphism groups in quantum physics. In: Govaerts, J., Hounkonnou, M.N., Msezane, A.Z. (eds.) Contemporary Problems in Mathematical Physics, Proceedings of the Third International Workshop, pp. 3-93. World Scientific, Hackensack, NJ, USA (2004)

4. Goldin, G.A.: Local current algebras: early ideas and new quantum-theoretical directions. Bulg. J. Phys. 33, 81-97 (2006)

5. Haubold, H.J., Mathai, A.M., Saxena, R.K.: Mittag-Leffler functions and their applications. J. Appl. Math. 2011, ID 298628 (2011)

6. Oliveira, M.J., Ouerdiane, H., Silva, J.L., Mendes, R.V.: The fractional Poisson measure in infinite dimensions. arXiv:1002.2124 
7. Sixdeniers, J.M., Penson, K.A., Solomon, A.I.: Mittag-Leffler coherent states. J. Physics A: Math. Gen. 32, 7543-7563 (1999)

8. Sirin, H., Ertik, H., Büyükkılıç, F., Demirhan, D.: Investigation of Bose-Einstein condensation based on fractality using fractional mathematics, J. Stat. Mech.: Theor. Exp. P10022 (2010)

9. Cipriano, F., Ouerdiane, H., Vilela, R.: Stochastic solution of a KPP-type nonlinear fractional differential equation. Fract. Calcul. Appl. Anal. 12, 47-57 (2009)

10. Mainardi, F., Gorenflo, R., Scalas, E.: A fractional generalization of the Poisson process. Vietnam J. Math. 32, 53-64 (2004)

11. Gelfand, I.M., Ya. Vilenkin, N.: Generalized Functions, vol. IV, Academic Press, New York, London (1968)

12. Albeverio, S., Kondratiev, Y., Röckner, M.: Analysis and geometry on configuration spaces. J. Funct. Anal. 154, 444-500 (1998)

13. Albeverio, S., Kondratiev, Y., Röckner, M.: Analysis and geometry on configuration spaces: the Gibbsian case. J. Funct. Anal. 157, 242-291 (1998)

14. Shimomura, H.: Poisson measures on the configuration space and unitary representations of the group of diffeomorphisms. J. Math. Kyoto Univ. 34, 599-614 (1994)

15. Parthasarathy, K.R.: Probability Measures on Metric Spaces. Academic Press, New York (1967)

16. Kondratiev, Y., Kuna, T.: Harmonic analysis on configuration space I. General theory. Infin. Dimens. Anal. Quantum Probab. Relat. Top. 5, 201-233 (2002)

17. Kondratiev, Y., Kuna, T., Oliveira, M.J.: Analytic aspects of Poissonian white noise analysis. Methods Funct. Anal. Topology 8, 15-48 (2002)

18. Kondratiev, Y., Kuna, T., Oliveira, M.J.: On the relations between Poissonian white noise analysis and harmonic analysis on configuration spaces. J. Funct. Anal. 213, 1-30 (2004)

19. T. Kuna; Studies in Configuration Space Analysis and Applications, $\mathrm{PhD}$ thesis, Bonner Mathematische Schriften Nr. 324, University of Bonn (1999)

20. Lenard, A.: Correlation functions and the uniqueness of the state in classical statistical mechanics. Comm. Math. Phys. 30, 35-44 (1973)

21. Lenard, A.: States of classical statistical mechanical systems of infinitely many particles I. Arch. Rational Mech. Anal. 59, 219-239 (1975)

22. Lenard, A.: States of classical statistical mechanical systems of infinitely many particles II. Arch. Rational Mech. Anal. 59, 241-256 (1975)

23. Fichtner, K.-H., Winkler, G.: Generalized Brownian motion, point processes and stochastic calculus for random fields. Math. Nachr. 161, 291-307 (1993)

24. M. J. Oliveira; Configuration Space Analysis and Poissonian White Noise Analysis, Ph. D. Thesis, Lisbon (2002). http://www.math.uni-bielefeld.de/igk/study-materials/corpo_2

25. Feller, W.: An introduction to probability theory and its applications, vol. John Wiley \& Sons, New York, II, Second Edition (1971)

26. Pollard, H.: The complete monotonic character of the Mittag-Leffler function $E_{\alpha}(-x)$. Bull. Amer. Math. Soc. 54, 1115-1116 (1948) 


\section{焦 Springer}

http://www.springer.com/978-3-319-16636-0

From Particle Systems to Partial Differential Equations II Particle Systems and PDEs II, Braga, Portugal, December 2013

Gonçalves, P.; Soares, A.J. (Eds.) 2015, VIII, 393 p. 29 illus., 10 illus. in color., Hardcover ISBN: $978-3-319-16636-0$ 